\title{
Microwave Spectra of Furazan. III. Rotation Spectra of Vibrationally Excited States
}

\author{
Otto L. Stiefvater \\ Coleg Prifysgol Gogledd Cymru, Bangor LL57 2 UW, Wales, U.K. \\ Z. Naturforsch. 45a, 1117-1130 (1990); received June 15, 1990 \\ Dedicated to Professor E. Bright Wilson, Jr. on the occasion of his 82. birthday
}

\begin{abstract}
The pure rotational spectra of molecules in 21 vibrationally excited states of the heterocyclic compound furazan $\left(\mathrm{C}_{2} \mathrm{H}_{2} \mathrm{~N}_{2} \mathrm{O}\right)$ have been detected and studied by DRM microwave spectroscopy. Rotational parameters are reported for the 12 fundamental levels below $1500 \mathrm{~cm}^{-1}$, and the contributions from 10 vibrational modes to the effective rotational constants and to the inertia defect of furazan are calculated.
\end{abstract}

\section{Introduction}

In two previous papers $[1 \mathrm{a}, \mathrm{b}]$ we have reported the doubly determined substitution structure [1 a] of the five-membered ring molecule furazan (1,2,5-oxadiazole, $\mathrm{C}_{2} \mathrm{H}_{2} \mathrm{~N}_{2} \mathrm{O}$ ). That structural information was needed before the electric field gradients around the quadrupolar nitrogen nuclei could be extracted from the hyperfine splittings of rotational transitions of the $3-\mathrm{d}_{1}$ form of this compound [1 b]. During the experimental work which was required for that structure determination, and which was carried out predominantly by double resonance modulation (DRM) microwave spectroscopy [1 c, d], [2], we could not avoid the detection of numerous vibrational satellite transitions in the pure rotation spectrum of furazan. Since the ${ }^{18} \mathrm{O}$-forms of normal and $\mathrm{d}_{2}$-furazan, with a natural isotopic abundance of only $0.2 \%$ of their parent forms, could be detected without much difficulty, we had to conclude that spectra of excited states with populations as small as $0.1 \%$ of the vibrational ground state (G.S.) would be detectable on the basis of the sensitivity of the DRM instrument. According to the Boltzmann law, this implied that the pure rotation spectra of all excited states up to vibrational energies of about $1500 \mathrm{~cm}^{-1}$ should be detectable.

Our decision to investigate these vibrational satellite spectra as fully as possible had several reasons of different weight:

Firstly, there is probably no safer way of preventing a weak satellite spectrum from being mistaken as the

Reprint requests to Dr. O. L. Stiefvater, Allgaierstraße 28, D-7512 Rheinstetten 2. ground state spectrum of a rare isotopomer than to assign that spectrum completely and then to examine the inertia defect for compatibility with that of the parent molecular form. Secondly, for the (then) planned quadrupole analysis on the $3-\mathrm{d}_{1}$ form [1 b] of furazan it seemed desirable to establish beforehand which hyperfine patterns would be falsified through overlap with vibrational satellite transitions of the normal or of the $\mathrm{d}_{2}$-form of furazan, which were both present ( $\sim 10 \%$ and $\sim 30 \%$, respectively) in the $3-\mathrm{d}_{1}$ sample. However, the prime reason for the present investigation was seen in the fact that the analysis of the spectra of vibrationally excited states has become a rather central theme of molecular spectroscopy during the past two decades. This field of research was first "ploughed" with the help of laser techniques, which yield the precise vibration frequencies and the molecular parameters of those excited states which happen to be accessible from the G.S. by use of a particular laser, and it has become a topic of high-resolution FT-IR spectroscopy during the past five years. In comparison with those approaches, the present study by DRM microwave techniques has the serious disadvantage of not being capable of yielding precise infrared frequencies. On the other hand, it promises the advantage of providing the rotational parameters not merely of one or two, but of all excited states up to the sensitivity limit of the DRM instrument. With only the Boltzmann population as the decisive factor, vibrational energy levels which cannot be reached from the G.S. by infrared techniques as a consequence of selection rules, are equally accessible to investigation by purely rotational spectroscopy as those levels which can be studied by infrared techniques. 


\section{Instrumental}

Experiments were carried out predominantly by DRM microwave spectroscopy, but measurements under Stark effect modulation (SEM) [3] were added when this appeared profitable. The DRM spectrometer [1 c] employed frequency modulation of the pump radiation. The modulation depth was variable from about $2 \mathrm{MHz}$ to $100 \mathrm{MHz}$ (upper limit dependent on klystron), and was usually kept near $30 \mathrm{MHz}$. For high sensitivity work the pump power emitted at the two frequencies was levelled through manual adjustment of the klystron supply voltages. The absorption cell of the instrument consisted of $20 \mathrm{~m}$ of "empty" $\mathrm{K}$-band waveguide. For the present work the range of pump frequencies was taken from $14.1 \mathrm{GHz}$ (cut-off frequency of K-band waveguide) up to $34 \mathrm{GHz}$, and the frequency of the signal radiation varied from $16 \mathrm{GHz}$ to $40 \mathrm{GHz}$. Both pump and signal klystrons were operated unstabilised. The latter was frequencyswept at rates of minimally $1 \mathrm{MHz} / \mathrm{sec}$ over ranges of, typically, $10 \mathrm{MHz}$ at a time. All frequency adjustments were carried out manually. Absorption signals were displayed on the oscilloscope, where their frequencies were measured to an estimated accuracy of $\pm 0.05 \mathrm{MHz}$. The SEM spectrometer was operated in an analogous fashion. All experiments were carried out at room temperature $(T \sim 300 \mathrm{~K})$ and at sample pressures near $10 \mathrm{mTorr}$.

\section{Spectroscopic Results}

\section{a) Rotational Assignments}

The identification of satellite spectra due to states with vibrational energies up to $1300 \mathrm{~cm}^{-1}$ was based on the observation, via double resonance (DR) techniques [ $1 \mathrm{c}, \mathrm{d}]$, of the pairs of Q-branch lines $4_{04} \rightarrow 4_{13} \rightarrow 4_{22}$. The signal transitions $4_{04} \rightarrow 4_{13}$ occur near $35 \mathrm{GHz}$, and the pump transitions $4_{13} \rightarrow 4_{22}$ fall into upper $\mathrm{K}$-band $(23-25 \mathrm{GHz})$. The result of a systematic examination of transitions in the vicinity of the G.S. pump line for DR signals near $35 \mathrm{GHz}$ is summarised in the double resonance map [1 c] of Figure 1. Similar maps result for the $J=5$ connections of the same series of transitions, and for many other sequences of satellite DRs between two Q-branch transitions of low $J$-value.

In progressing to states with vibrational energies above $1300 \mathrm{~cm}^{-1}$, and hence with still smaller Boltz-

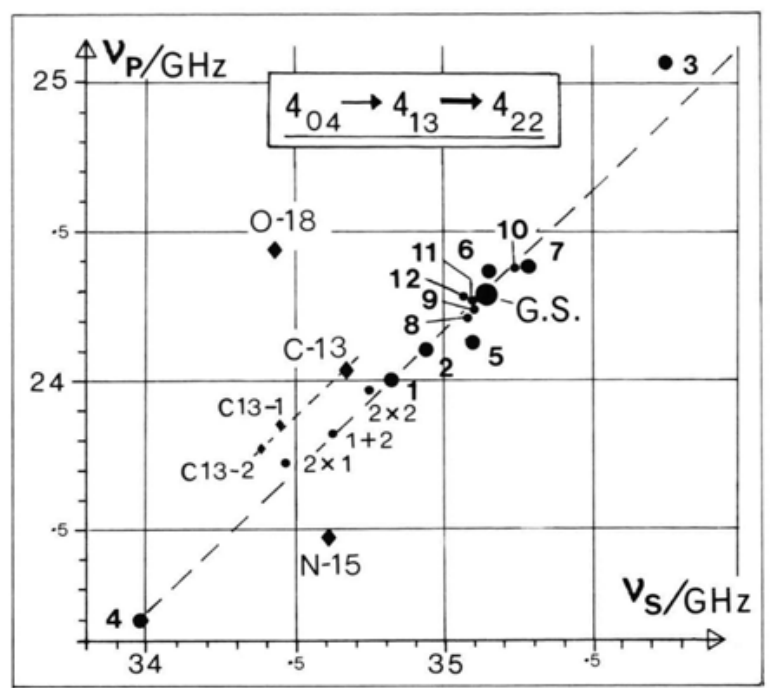

Fig. 1. DR-map of the connection $4_{04} \rightarrow 4_{13} \rightarrow 4_{22}$, illustrating the displacement from the G.S. of transitions due to excited vibration states and isotopomers of furazan. (The numbering 1 through 12 indicates the decreasing intensity of satellites. Accurate frequencies of transitions are given in the Appendix.)

mann populations, the decreasing intensity of rotational lines of low $J$-value demanded a switch from the $J=4$ connection to a DR scheme that consisted of more intense rotational transitions. Accordingly, the $J=14$ connections were chosen which consist of the three transitions $14_{9,5} \rightarrow 14_{10,4} \rightarrow 14_{11,3} \rightarrow 14_{12,2}$ at $\sim 37 \mathrm{GHz}, \sim 25 \mathrm{GHz}$ and $\sim 30 \mathrm{GHz}$, respectively, with the $\mathrm{K}$-band line near $25 \mathrm{GHz}$ as common pump transitions for the two signals. While the pump transitions lie at the band head of the series $J_{J-4,4} \rightarrow J_{J-3,3}$, with the consequence that their frequencies vary relatively little from the G.S. for different excited states, the signal frequencies for that scheme vary by considerably larger displacements from the G.S. than those of the $J=4$ connections of Fig. 1. The identified DR-connections are presented as Table 1 . The various vibrationally excited states which give rise to these rotational transitions are designated in that table by numbers ' 1 ' through ' 12 ' in order of decreasing intensity of the signals (increasing vibrational energy). The transitions of the isotopomers are included in that table since their intensity (due to natural abundance) provided a first clue on the vibrational energy of identified states. The notation " $2 \times 1$ " in the table and in Fig. 1 indicates that state ' 1 ' is excited by two quanta (overtone level), while " $2+1$ " designates the state in 
Table 1. Frequencies (in MHz) of DR-connected transitions between $J=14$ levels in furazan.

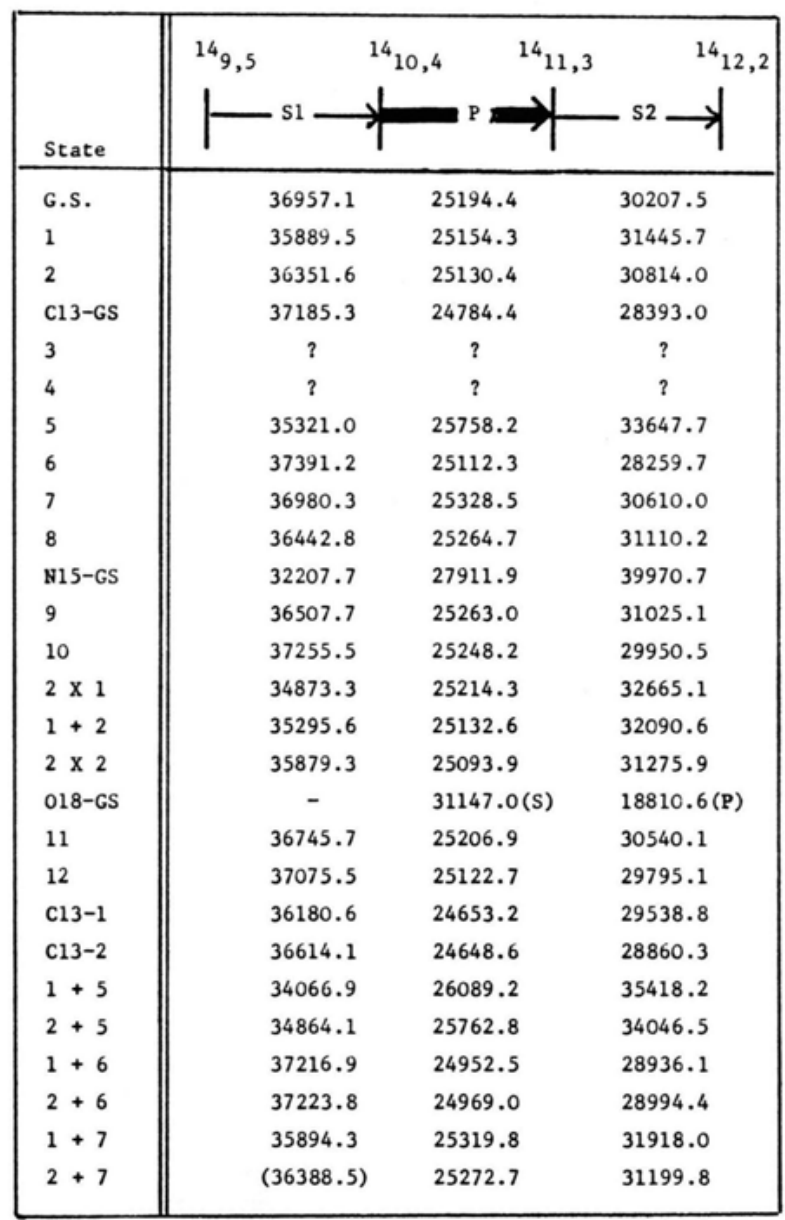

which mode ' 1 ' and mode ' 2 ' are simultaneously excited by one quantum each (combination level). The correlation with IR-designations is given at the bottom of Table 2 and across the top of Figure 2.

Upon inspection of Fig. 1 or Table 1 the informed reader will realise that every DR connection (marked by a circle in Fig. 1), and even more so the identification of two signal transitions which arise from the same pump transition (i.e. every row in Table 1), establishes the entire Q-branch of one vibrational satellite spectrum. - With the Q-branches of satellite spectra established by these experiments and further refined through the measurements of more, but predictable DRs within each Q-branch, the $J=2$ and $J=3$ members of each satellite (which had either been measured or which were precisely predictable) were chosen as the pump transitions for the $J=1 \rightarrow 2$ and $J=2 \rightarrow 3$ R-branch transitions. The successful completion of these DR experiments yielded the rotational constants of satellites up to $1600 \mathrm{~cm}^{-1}$ of vibrational energy.

Fairly precise rotational constants were derived from least-squares fits of the spectra after all accessible transitions (between 80 and 95 lines for each of the fundamentals) had been measured by either DRM or SEM techniques along with the work on the isotopic forms [1 a]. The overall results, together with statistical information on each spectrum, are collected in Table 2. - Although a total of 21 vibrational satellite spectra has been observed and assigned (see Fig. 2), Table 2 lists the rotational parameters of the G.S. and the 12 fundamentals only. The identified transitions of each of those 13 spectra are given as Appendix.

\section{b) Perturbations in the Rotational Satellite Spectra}

The rotational spectrum of the G.S. was readily fitted up to $J=24$ to a Hamiltonian with four quartic distortion constants [ $1 \mathrm{a}$ ], and the same behaviour was expected for the vibrational satellite spectra. For six spectra, labelled ' 7 '-'12' in figures and tables, this was indeed the case, and the distortion constants for these six spectra are qualitatively similar to those determined for the G.S.

For the satellites arising from states with vibrational energies between $600 \mathrm{~cm}^{-1}$ and $900 \mathrm{~cm}^{-1}$ several incompatibilities with the simple quartic Hamiltonian were observed: In the most intense spectrum (labelled '1') it was found that some transitions above $J=13$ showed deviations from rigid behaviour which increased to $\sim 26 \mathrm{MHz}$ at $J=23$. These DR-identified perturbed transitions (given in italics in the Appendix) were not included in the final LSQ-fit which from 80 well-behaved transitions yielded rotational constants with $3 \sigma$-uncertainties of $0.03 \mathrm{MHz}$. Similar, though smaller, deviations occur for transitions with $J=$ $18-22$ in the second most intense satellite spectrum '2'. Deviations from rigid rotor behaviour occur also in the spectra labelled ' 5 ' and ' 6 '. They set in at $J=15$ to reach about $16 \mathrm{MHz}$ in the case of satellite ' 5 ', and at $J=11$ to reach about $13 \mathrm{MHz}$ in satellite spectrum ' 6 '. The fitted transitions of those two satellites also lead to abnormally large values of opposite signs for the distortion constants $\tau_{a a b b}$ and $\tau_{a b a b}$ (see rows 10 and 11 in Table 2).

Much larger deviations from the rigid rotor pattern than in those four spectra are encountered in the spec- 
Table 2. Rotational parameters of the G.S. and 12 vibrationally excited states of furazan.

\begin{tabular}{|c|c|c|c|c|c|c|c|c|}
\hline 1 & Satellite spectrum & G.s. & 1 & 2 & 3 & 4 & 5 & 6 \\
\hline $\begin{array}{l}2 \\
3 \\
4\end{array}$ & $\begin{array}{l}\text { Observed transitions } \\
\text { Range of J-values } \\
\left.\text { St'd dev'n }(\mathrm{MHz}),\left(\mathrm{N}_{\mathrm{f}}\right)^{\mathrm{a}}\right)\end{array}$ & $\begin{array}{l}12 R \& 82 Q \\
1-8 \& 2-24 \\
0.055(94)\end{array}$ & $\begin{array}{l}6 R \& 86 Q \\
1-3 \& 2-23 \\
0.051(80)\end{array}$ & $\begin{array}{l}6 R \& 85 Q \\
1-3 \& 2-23 \\
0.052(86)\end{array}$ & $\begin{array}{l}6 \text { R } \& 70 Q \\
1-3 \& 2-13 \\
7.5(9)\end{array}$ & $\begin{array}{l}6 \text { R } \& 46 Q \\
1-3 \& 2-10 \\
4.5 \text { (9) }\end{array}$ & $\begin{array}{l}6 R \& 81 Q \\
1-3 \& 2-23 \\
0.072(73)\end{array}$ & $\begin{array}{l}5 R \& 88 Q \\
1-3 \& 2-24 \\
0.071(76)\end{array}$ \\
\hline 5 & $A$ & $10457.21(2)^{b)}$ & $10427.54(3)$ & $10436.27(3)$ & $10395.8(16)$ & $10410.1(10)$ & $10476.46(3)$ & $10431.52(3)$ \\
\hline 6 & in $\mathrm{MHz}$ & $9681.65(2)$ & $9624.04(3)$ & $9647.14(3)$ & $9919.1(18)$ & $9407.8(10)$ & $9621.18(3)$ & $9703.98(3)$ \\
\hline 7 & $c$ & $5023.70(2)$ & $5023.69(2)$ & $5025.11(2)$ & $5044.4(8)$ & $4996.6(5)$ & $5001.55(3)$ & $5024.56(3)$ \\
\hline 8 & $\tau_{a a a a}$ & $-17.1(6)$ & $-20.0(48)$ & $-17.5(48)$ & - & - & $-46.3(70)$ & $+7.3(68)$ \\
\hline 9 & $\tau_{b b b b}$ & $-20.5(6)$ & $-23.0(46)$ & $-20.8(45)$ & - & - & $-23.4(70)$ & $-18.7(63)$ \\
\hline 10 & $\tau^{\top} a a b b$ & $-4.4(20)$ & $-16.3(141)$ & $-5.7(141)$ & - & - & $-133.3(213)$ & $+128.0(204)$ \\
\hline 11 & $\tau_{a b a b}$ & $-7.8(7)$ & $-4.6(48)$ & $-6.4(48)$ & - & - & $+44.4(72)$ & $-56.4(69)$ \\
\hline 12 & $a_{A}=A_{\text {G.S. }}-A_{V}$ & - & $+29.67(3)^{c)}$ & $+20.94(3)$ & +61.4 & +47.1 & $-19.25(3)$ & $+25.69(3)$ \\
\hline 13 & $\alpha_{B}=B_{G . S .}-B_{V}$ & - & $+57.61(3)$ & $+34.51(3)$ & -237.5 & +273.8 & $+60.47(3)$ & $-22.33(3)$ \\
\hline 14 & ${ }^{a} C=c_{G . S .}-c_{V}$ & - & $+0.01(2)$ & $-1.41(2)$ & -20.7 & +27.1 & $+22.15(3)$ & $-0.86(3)$ \\
\hline 15 & $I_{a} \quad T$ & $48.3280(1)$ & $48.4655(1)$ & $48.4250(1)$ & $48.613(7)$ & $48.547(5)$ & $48.2392(2)$ & $48.4470(2)$ \\
\hline 16 & in $u R^{2}$ & $52.1994(1)$ & $52.5118(2)$ & $52.3861(2)$ & $50.950(9)$ & $53.719(6)$ & $52.5274(2)$ & $52.0792(2)$ \\
\hline 17 & $I_{c} \quad J$ & $100.5983(3)$ & $100.5985(4)$ & $100.5702(4)$ & $100.185(15)$ & $101.145(9)$ & $101.0439(5)$ & $100.5812(5)$ \\
\hline 18 & I.D. $=I_{c}-I_{a}-I_{b}$ & $+0.0709(3)$ & $-0.3788(4)$ & $-0.2408(4)$ & $+0.622(19)$ & $-1.121(12)$ & $+0.2772(6)$ & $+0.0550(6)$ \\
\hline 19 & $\delta(I D)=I D_{V}-I D_{G_{0} . S}$ & - & $-0.4497(4)^{c)}$ & $-0.3117(4)$ & $+0.551(19)$ & $-1.192(12)$ & $+0.2063(6)$ & $-0.0159(6)$ \\
\hline $\begin{array}{l}20 \\
21\end{array}$ & $\begin{array}{l}\text { Species/frequ'y }\left(\mathrm{cm}^{-1}\right) \\
\text { in-p1ane/out-of-plane }\end{array}$ & & $\begin{array}{l}\mathrm{B}_{2} / 631 \\
\text { out }\end{array}$ & $\begin{array}{l}\mathrm{A}_{2} / 635 \\
\text { out }\end{array}$ & $\begin{array}{l}\mathrm{B}_{1} / 820 ? \\
\text { in ? }\end{array}$ & $\begin{array}{c}\mathrm{B}_{2} / 838 ? \\
\text { out ? }\end{array}$ & $\mathrm{A}_{1} / 872$ & $\begin{array}{l}\mathrm{A}_{2} / 888 \\
\text { out }\end{array}$ \\
\hline 22 & I.R. mode , level & & $v_{15}=1$ & $v_{8}=1$ & $v_{13}=1 ?$ & $v_{14}=1 ?$ & $v_{6}=1$ & $v_{7}=1$ \\
\hline
\end{tabular}

\begin{tabular}{|c|c|c|c|c|c|c|c|}
\hline 1 & Satellite spectrum & 7 & 8 & 9 & 10 & 11 & 12 \\
\hline $\begin{array}{l}2 \\
3 \\
4\end{array}$ & $\begin{array}{l}\text { Observed transitions } \\
\text { Range of J-values } \\
\text { St'd dev'n (MHz), }\left(\mathrm{N}_{\mathrm{f}}\right)^{\text {a) }}\end{array}$ & $\begin{array}{l}6 R \& 85 Q \\
1-3 \& 2-23 \\
0.050(91)\end{array}$ & $\begin{array}{l}6 R \& 83 Q \\
1-3 \& 2-23 \\
0.056(89)\end{array}$ & $\begin{array}{l}6 R \& 83 Q \\
1-3 \& 2-23 \\
0.058(89)\end{array}$ & $\begin{array}{l}5 R \& 82 Q \\
2-3 \& 3-24 \\
0.059(87)\end{array}$ & $\begin{array}{l}5 R \& 81 Q \\
2-3 \& 3-24 \\
0.056(86)\end{array}$ & $\begin{array}{l}5 R \& 73 Q \\
2-3 \& 3-24 \\
0.056(78)\end{array}$ \\
\hline 5 & $A$ & $10471.51(2)$ & $10465.43(3)$ & $10468.79(3)$ & $10465.88(3)$ & $10447.68(3)$ & $10433.55(3)$ \\
\hline 6 & in $\mathrm{MHz}$ & $9686.22(2)$ & $9669.03(3)$ & $9674.32(3)$ & $9696.14(3)$ & $9664.37(3)$ & $9667.85(3)$ \\
\hline 7 & $c$ & $5011.15(2)$ & $5027.39(2)$ & $5029.51(2)$ & $5019.19(2)$ & $5014.52(2)$ & $5013.81(2)$ \\
\hline 8 & $\tau^{\tau} a a a a$ & $-18.1(43)$ & $-22.5(49)$ & $-25.6(51)$ & $-16.3(52)$ & $-27.4(50)$ & $-22.2(51)$ \\
\hline 9 & ${ }^{\top} b b b b$ & $-20.9(40)$ & $-25.1(47)$ & $-28.7(48)$ & $-19.7(49)$ & $-28.7(48)$ & $-27.2(48)$ \\
\hline 10 & $\tau_{a a b b}$ & $-4.8(130)$ & $-19.2(149)$ & $-31.6(153)$ & $-1.2(156)$ & $-36.8(150)$ & $-22.0(153)$ \\
\hline 11 & ${ }^{\top} a b a b$ & $-8.1(45)$ & $-3.0(49)$ & $+1.0(51)$ & $-8.8(52)$ & $+3.6(50)$ & $-2.2(51)$ \\
\hline 12 & $a_{A}=A_{G . S .}-A_{V}$ & $-14.30(2)$ & $-8.22(3)$ & $-11.58(3)$ & $-8.67(3)$ & $+9.53(3)$ & $+23.66(3)$ \\
\hline 13 & $a_{B}=B_{G . S .}-B_{V}$ & $-4.57(2)$ & $+12.62(3)$ & $+7.33(3)$ & $-14.49(3)$ & $+17.28(3)$ & $+13.80(3)$ \\
\hline 14 & $a_{C}=c_{G . S .}-c_{V}$ & $+12.55(2)$ & $-3.69(2)$ & $-5.81(2)$ & $+4.51(2)$ & $+9.18(2)$ & $+9.89(2)$ \\
\hline 15 & ( 2 d) & $48.2620(1)$ & $48.9201(1)$ & $48.2745(2)$ & $48.2879(2)$ & $48.3721(2)$ & $48.4376(2)$ \\
\hline 16 & In wh & $52.1747(1)$ & $52.2675(2)$ & $52.2389(2)$ & $52.1214(2)$ & $52.2927(2)$ & $52.2739(2)$ \\
\hline 17 & $I_{c}$ & $100.8502(3)$ & $100.5246(4)$ & $100.4821(4)$ & $100.6888(4)$ & $100.7825(4)$ & $100.7969(4)$ \\
\hline 18 & I.D. $=I_{a}-I_{a}-I_{b}$ & $+0.4135(4)$ & $-0.0330(5)$ & $-0.0314(5)$ & $+0.2795(5)$ & $+0.1177(5)$ & $+0.0854(5)$ \\
\hline 19 & $\delta(I D)=I D_{V}-I D_{G . S .}$ & $+0.3426(4)$ & $-0.1039(5)$ & $-0.1023(5)$ & $+0.2086(5)$ & $+0.0468(5)$ & $+0.0145(5)$ \\
\hline 20 & Species/frequ'y $\left(\mathrm{cm}^{-1}\right)$ & $B_{1} / 952$ & $\mathrm{~A}_{1} / 1005$ & $A_{1} / 1036$ & $B_{1} / 1175$ & $A_{1} / 1316$ & $A_{1} / 1418$ \\
\hline 21 & in-plane/out-of-plane & in & in & in & in & in & in \\
\hline 22 & I.R. mode, level & $v_{12}=1$ & $v_{5}=1$ & $v_{4}=1$ & $v_{11}=1$ & $v_{3}=1$ & $v_{2}=1$ \\
\hline
\end{tabular}

a) $N_{f}$ is the nmber of transitions in the LSQ fit. - ${ }^{\text {b) }}$ All uncertainties are $3 \sigma$-values, and given in units of the last digit. c) Uncertainties in the G.S. parameters have been ignored when differences were taken. $-{ }^{\text {d) }}$ Conversion factor: $505376 \mathrm{u} \AA^{2} \mathrm{MHz}$. 
tra labelled ' 3 ' and ' 4 '. In these two satellite spectra even the transitions with the lowest $J$-value do not conform with rigid rotor expectation. A selection of 9 transitions with $J \leqq 3$ allowed these deviations to be kept below $10 \mathrm{MHz}$ in both cases and permitted the rotational constants to be determined to $\pm 2 \mathrm{MHz}$. These constants, together with the strongly perturbed positions of absorption lines in those two spectra, point to the presence of Coriolis coupling [4] between these two vibrational modes. This throws the rotational constants $B$ some $\pm 250 \mathrm{MHz}$ off the otherwise expected values. The effect of this coupling on the rotational transition frequencies is such that it necessitated the identification of every transition through DR-double-search [1 c] experiments or, in the absence of a common energy level between a pair of transitions, through the observation of collisional relaxation effects under DRM [1 e]. Despite the time-consuming nature of such work, we have carried out such experiments up to transitions with $J=13$ in the case of satellite ' 3 ', and up to $J=10$ for satellite ' 4 '. At these $J$-values, the deviations of transition frequencies from expectations based on a rigid rotor prediction reach up to $10 \mathrm{GHz}$, with the consequence that it became impossible to predict with any confidence whether transitions with higher $J$-values would still fall within the frequency range of the DRM instrument. For this reason we have not tried to investigate these two spectra any further. We would suggest that the data in columns 3 and 4 of Table 2 be taken as merely qualitative, with little weight attachable to the error limits quoted there.

\section{c) Correlation of Satellite Spectra with Excitation of Normal Modes of Vibration}

As far as we are aware, there exists no theory which, in the absence of detailed knowledge about the molecular force field, would allow a correlation of the observed changes in the effective moments of inertia (or rotational constants) with the excitation of a specific normal mode of vibration in a molecule. One is therefore restricted to forging the link between rotational and vibrational spectroscopy almost exclusively on the basis of a comparison of the vibrational exitation energies which are obtained in both these two fields of molecular spectroscopy.

The vibrational spectrum of furazan has been the subject of several investigations [5-8] by infrared spectroscopy. A complete assignment of the funda- mental frequencies of normal modes of vibration has been given by Christensen et al. [8] for normal and deuterated furazan. These researchers quote an accuracy of $\pm 1 \mathrm{~cm}^{-1}$ for their frequency determinations, that is: for the energy of vibrational excitation from the G.S. The following correlation of satellites in the pure rotational spectrum with vibrationally excited fundamenal, overtone and combination levels rests entirely on their work.

As is generally known, the determination of vibrational energies (expressed in wavenumber units) by microwave techniques is accomplished through measurements of the intensity of a satellite spectrum relative to the intensity of rotational transitions associated with molecules in the vibrational G.S. From such intensity ratios the vibrational energy separation between a satellite and the G.S. may be deduced via the Boltzmann law. The accuracy which can be achieved in such microwave experiments is significantly inferior to that of corresponding data from IR spectroscopy. It ranges generally from about $\pm 3 \%$ (optimally) to $\pm 20 \%$.

In furazan such vibrational energy determinations via "relative intensity measurements" [9] are, - initially -, hindered by the fact that the $\mathrm{C}_{2 \mathrm{v}}$ symmetry of the molecule, together with the occurrence of a pair of equivalent nitrogen nuclei $\left(I_{\mathrm{N}}=1\right.$, bosons) and a pair of equivalent protons $\left(I_{\mathbf{H}}=1 / 2\right.$, fermions), has a pronounced influence on the intensity of all rotational transitions. However, if these "nuclear spin statistical weight" effects within each satellite spectrum are analysed first, they offer the possibility of ascertaining whether a particular spectrum arises from molecules in an excited state of a symmetric (A-species) or an antisymmetric (B-species) mode of vibration. Furthermore, if that information is combined with the value of the inertia defects (I.D.), which emerge from the rotational analysis and which usually provide a strong hint on whether a particular satellite arises from excitation of an "in-plane" or of an "out-of-plane" vibration, it becomes possible to distinguish between the four symmetry species $A_{1}, A_{2}, B_{1}$ and $B_{2}$ of the $\mathrm{C}_{2 \mathrm{v}}$ group, into which the various excited vibration states of furazan must fall. Subsequent intensity measurements on satellite transitions relative to the G.S. and other satellites under proper consideration of spin weights will then allow a complete correlation of excited state energies with known IR frequencies.

As detailed in the following two subsections, the experimental procedures resulting from these con- 
siderations were successfully applied to the satellite spectra of furazan, and produced a satisfactory correlation of the present rotational data with information from the IR-study of Christensen et al. [8] (see Table 5).

\section{c.1. Symmetry, Nuclear Spin Weights and Intensities $[10,11]$}

Consider the total wavefunction of the molecule which, under omission of the electronic factor, may be written as

$$
\Psi_{\text {total }}=\Psi_{\text {vib. }} \times \Psi_{\text {rot. }} \times \Psi_{\text {spin }},
$$

where $\Psi_{\text {vib. }}=\prod_{i} \psi_{n_{i}}^{(i)}\left(Q_{i}\right)$ is the vibrational wavefunction for a state in which the modes $i$, described by normal coordinates $Q_{i}$, are excited by $n_{i}$ quanta. Under the symmetry operation $\mathrm{C}_{2}$ this part of $\Psi_{\text {total }}$ transforms like the normal coordinate $Q_{i}[10]$, while the rotational factor $\Psi_{\text {rot. }}\left(J, K_{-}, K_{+}\right)$is even or odd under $\mathrm{C}_{2}$ in accordance with even or odd values of $\left(K_{-}+K_{+}\right)[11]$. As the interchange of the two protons of furazan requires $\Psi_{\text {total }}$ to be antisymmetric (odd) under $\mathrm{C}_{2}$, it follows that antisymmetric spin functions have to be combined with even rotational functions when the vibrational factor, $\Psi_{\text {vib. }}$, is even (A-species). Conversely, if the normal mode $i$ (and its wavefunction) is antisymmetric (odd) under the $\mathrm{C}_{2}$ operation, the product $\Psi_{\text {rot. }} \times \Psi_{\text {spin }}$ must be even. This requires the rotational part and the spin factor to have the same symmetry.

The nuclear spin function, $\Psi_{\text {spin }}$, itself may be any one of the $\left(2 I_{\mathrm{N}}+1\right)^{2} \times\left(2 I_{\mathrm{H}}+1\right)^{2}=9 \times 4=36$ products of the form

$$
\Psi_{\text {spin }}=\psi_{\mathrm{s}}(2 \mathrm{~N}) \times \psi_{\mathrm{s}}(2 \mathrm{H}),
$$

where $\psi_{\mathrm{s}}(2 \mathrm{~N})$ and $\psi_{\mathrm{s}}(2 \mathrm{H})$ represent the degenerate spin states resulting from the combination of two nitrogen spins or two proton spins, respectively. Of the 36 spin functions above,

$\left\{\left(2 I_{\mathrm{N}}+1\right)^{2} \times\left(2 I_{\mathrm{H}}+1\right)^{2}+\left(2 I_{\mathrm{N}}+1\right) \times\left(2 I_{\mathrm{H}}+1\right)\right\} / 2=21$

are symmetric (even) under the operation $\mathrm{C}_{2}$, the remainder of 15 being antisymmetric (odd) [11].

If these nuclear spin weights are introduced into the symmetry consideration above, one obtains the relative weights as given in Table 3 for even and odd rotational transitions depending on the symmetry of the vibrational mode excited. Hence, the symmetry of $\Psi_{\text {vib. }}$ and of $\psi_{n_{i}}^{(i)}\left(Q_{i}\right)$ can be determined experimen-
Table 3. Nuclear spin weights for rotational transitions associated with even or odd excited vibrational levels in furazan.

\begin{tabular}{lll}
\hline $\begin{array}{l}\text { Symmetry of } \\
\text { vibrational } \\
\text { wavefunction }\end{array}$ & $\begin{array}{l}\text { Rotational } \\
\text { symmetry } \\
\left(K_{-}+K_{+}\right)\end{array}$ & Spin weight \\
\hline$\Psi_{\text {vib. }}=$ even & even & 5 \\
$\Psi_{\text {vib. }}=$ odd & odd & 7 \\
\hline
\end{tabular}

tally from a comparison of the intensity of an even rotational transition with that of an odd one within the satellite spectrum associated with excitation of the mode $i$.

\section{c.2. Experimental Results}

Relative intensity measurements for the determination of the vibrational symmetries were carried out on most excited vibration state spectra of furazan. One example, reproduced as Table 4, may suffice to illustrate the details:

Three pairs of even and odd rotational transitions of the satellite ' 2 ' with rotational frequency separations of about $20 \mathrm{MHz}$ were selected to ensure a flat response of the SEM spectrometer. Each transition was cross-checked against coincidence with a transition from the other 26 spectra associated with the sample, since overlap would, clearly, falsify the apparent intensity. The intensity ratio between the rotationally even and odd component of each pair was then determined experimentally (last column of Table 4). The theoretical intensity of each transition, which had been pre-calculated without consideration of the spin weights (column $\mathrm{V}$ of the table), was then multiplied with the spin weights that would apply if state ' 2 ' corresponded with a symmetric (even) or antisymmetric (odd) vibration, and the expected intensity ratios (columns VIb, VII b) were formed for both possibilities. - As may be verified from the experimental results in column VIII, there could not remain any doubt that state ' 2 ' arises from excitation of a symmetric vibration. The inertia defect contribution $\delta(\mathrm{ID})=-0.3117(4) \mathrm{u} \AA^{2}$ (see Table 2 or 5) suggests that state ' 2 ' arises from an "out-of-plane" vibration, which together with the later determined energy separation of $\sim 650 \mathrm{~cm}^{-1}$ from the G.S. left little choice but to identify state ' 2 ' with excitation of the IR-inactive fundamental $v_{8}$ at $635 \mathrm{~cm}^{-1}$. 
Table 4. Symmetry determination of satellite '2'.

\begin{tabular}{|c|c|c|c|c|c|c|c|c|}
\hline I & II & III & VI & V & VIa & vIb & VIIa VIIb & VIII \\
\hline Pair & $\begin{array}{l}\text { Transition } \\
\text { frequency } \\
\text { (in MHz) }\end{array}$ & $\begin{array}{l}\text { Rotational } \\
\text { assignment }\end{array}$ & $\begin{array}{l}\text { Rotational } \\
\text { symmetry } \\
\left(\mathrm{K}_{+}+\mathrm{K}_{-}\right)\end{array}$ & $\begin{array}{l}\text { Intensity } \\
\text { wi thout } \\
\text { weights }\end{array}$ & \multicolumn{3}{|c|}{$\begin{array}{l}\text { Weights \& intensity ratio for } \\
\psi_{1 \text {, vib. }}^{(2)}=\text { even } \mid \psi_{1, \text { vib. }}^{(2)}=\text { odd }\end{array}$} & $\begin{array}{l}\text { Observed } \\
\text { intensity } \\
\text { ratio }\end{array}$ \\
\hline 1 & $\begin{array}{l}21251.6 \\
21258.2\end{array}$ & $\begin{array}{l}5_{4,2}-5_{5,1} \\
7_{6,1}-7_{7,0}\end{array}$ & $\begin{array}{l}\text { even } \\
\text { odd }\end{array}$ & $\begin{array}{l}22.54 \\
28.30\end{array}$ & $\begin{array}{l}5 \\
7\end{array}$ & 0.569 & 1.115 & 0.58 \\
\hline 2 & $\begin{array}{l}28833.3 \\
28851.8\end{array}$ & $\begin{array}{l}11_{9,2}-11_{10,1} \\
1_{12,4}-16_{13,3}\end{array}$ & $\begin{array}{l}\text { odd } \\
\text { even }\end{array}$ & $\begin{array}{l}50.44 \\
91.23\end{array}$ & $\begin{array}{l}7 \\
5\end{array}$ & 0.774 & 0.395 & 0.74 \\
\hline 3 & $\begin{array}{l}30795.1 \\
30814.0\end{array}$ & $\begin{array}{c}{ }^{9} 8,1-{ }^{9} 9,0 \\
{ }^{14} 11,3^{-14} 12,2\end{array}$ & $\begin{array}{l}\text { odd } \\
\text { even }\end{array}$ & $\begin{array}{l}25.63 \\
70.28\end{array}$ & $\begin{array}{l}7 \\
5\end{array}$ & 0.510 & $\begin{array}{l}5 \\
7\end{array}$ & 0.50 \\
\hline
\end{tabular}

Table 5. Summary of results of intensity measurements on satellite spectra of furazan and correlation with IR data [8].

\begin{tabular}{|c|c|c|c|c|c|c|}
\hline \multicolumn{4}{|c|}{ MICROWAVE } & \multicolumn{3}{|c|}{ INFRARED } \\
\hline $\begin{array}{l}\text { Lesig- } \\
\text { nation }\end{array}$ & $\begin{array}{l}\text { Symetry } \\
\text { of } \mathbf{\Psi}_{\mathrm{vib}}\end{array}$ & $\begin{array}{l}\text { Vibrat' } 1 \\
\text { energy } \\
\left(\text { in } \mathrm{cm}^{-1}\right)\end{array}$ & $\begin{array}{c}\delta(\mathrm{ID}) \\
\left(\text { in } u \mathcal{R}^{2}\right)\end{array}$ & $\begin{array}{l}\text { Desig- } \\
\text { nation }\end{array}$ & $\begin{array}{c}\text { Energy } \\
\left(\text { in } \mathrm{cm}^{-1} \text { ) }\right.\end{array}$ & $\begin{array}{l}\text { Syrmetry } \\
\text { species }\end{array}$ \\
\hline 1 & odd & $\sim 650$ & $-0.4497(4)$ & $v_{15}$ & 631 & $\mathrm{~B}_{2}$ \\
\hline 2 & even & & $-0.3117(4)$ & $v_{8}$ & 634 & $A_{2}$ \\
\hline 3 & $?$ & $?$ & $+0.551 ?$ & $v_{13}$ & 820 & $B_{1}$ \\
\hline 4 & $?$ & $?$ & $-1.192 ?$ & $v_{14}$ & 838 & $\mathrm{~B}_{2}$ \\
\hline 5 & even & $\sim 880$ & $+0.2063(6)$ & $v_{6}$ & 872 & $\mathbf{A}_{1}$ \\
\hline 6 & even & & $-0.0159(6)$ & $v_{7}$ & 888 & $A_{2}$ \\
\hline 7 & odd & $\sim 950$ & $+0.3426(4)$ & $v_{12}$ & 953 & $B_{1}$ \\
\hline 8 & even & $\sim 1000$ & $-0.1039(5)$ & $v_{5}$ & 1005 & $A_{1}$ \\
\hline 9 & even & $\sim 1040$ & $-0.1023(5)$ & $v_{4}$ & 1036 & $\mathbf{A}_{1}$ \\
\hline 10 & odd & $\sim 1190$ & $+0.2086(5)$ & $v_{11}$ & 1175 & $B_{1}$ \\
\hline 11 & even & 21310 & $+0.0468(5)$ & $v_{3}$ & 1316 & $A_{1}$ \\
\hline 12 & even & $\sim 1420$ & $+0.0145(5)$ & $v_{2}$ & 1418 & $\mathbf{A}_{1}$ \\
\hline
\end{tabular}

Note: The negative inertia defect contribution of the "inplane" vibrations 8 and $9\left(v_{5}\right.$ and $\left.v_{4}\right)$ should be noted.

Naturally, it became more difficult with increasing vibrational energy to find suitable pairs of odd and even transitions for intensity comparison within each satellite spectrum. However, at least two such pairs were examined for every fundamental level, and the fact that the relative intensity ratios would have to be in error by a factor $49 / 25 \sim 2$ to result in an incorrect vibrational symmetry greatly enhances our confidence in this part of the work.

No such symmetry determinations were attempted on states ' 3 ' and ' 4 ' as it was obvious that a great deal of "intensity borrowing" was occurring within their mutually perturbed spectra.

Following the determination of vibrational symmetries of satellites, their energy separations from the G.S. and from each other were obtained from further relative intensity measurements, in which the now known weight factors were properly taken into account. While intensity ratios involving G.S. transitions had not sufficient accuracy for distinguishing with certainty between two states that lie within less than $30 \mathrm{~cm}^{-1}$ of each other, intensity comparisons between such states allowed a definite conclusion as to which one was higher in energy, including a qualitative determination of the vibrational energy gap between them.

The overall results of relative intensity measurements on vibrational satellite spectra in furazan are collected in Table 5. The left half of this table gives the findings from the present microwave work for comparison and correlation with the IR data [8], which are given in the right half of the table.

\section{d) Summary of Microwave Results}

A comprehensive illustration of the present work on the pure rotation spectra of vibrationally excited states of furazan is attempted in Figure 2. In this dia- 


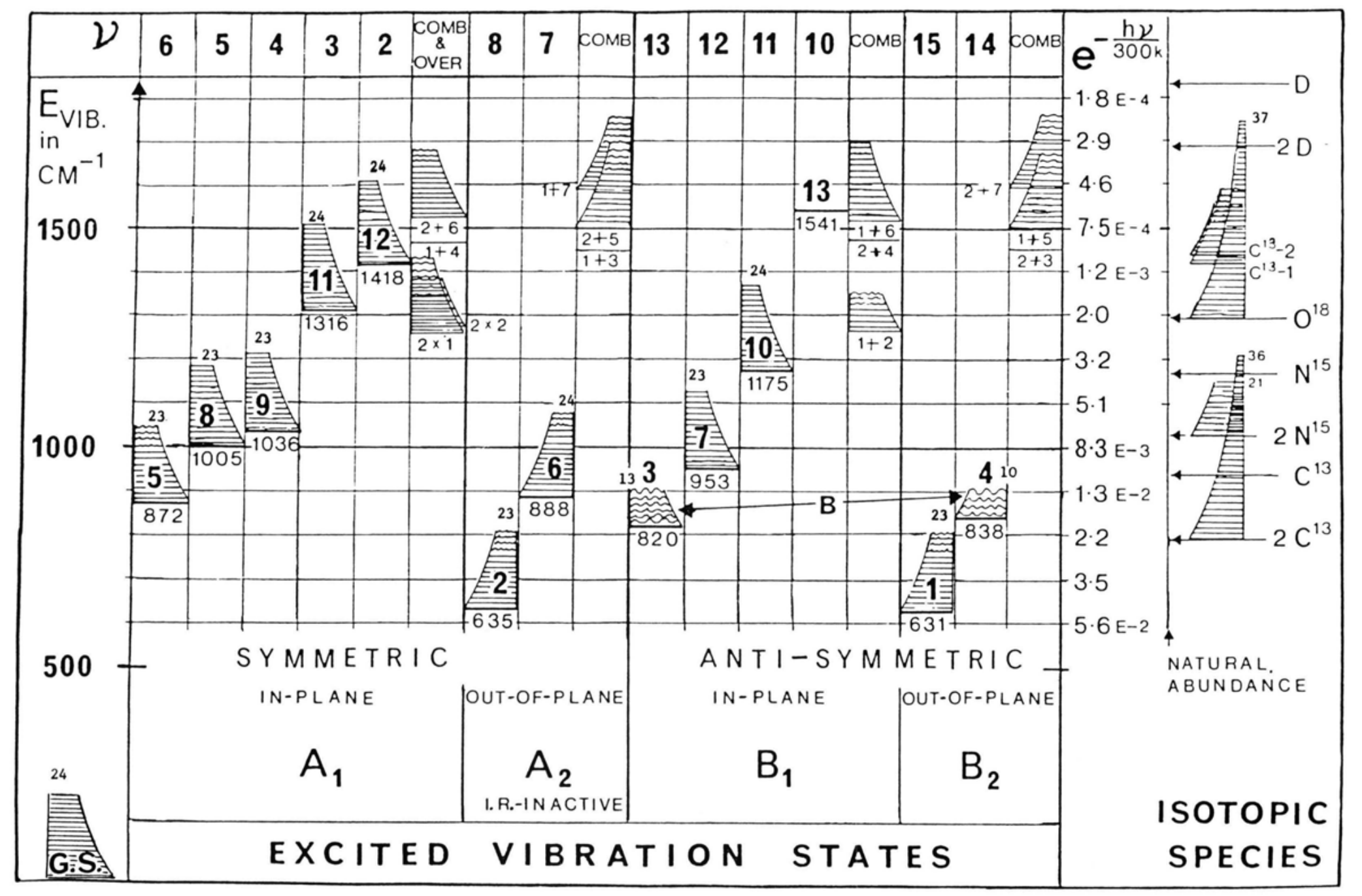

Fig. 2. Illustration of vibrationally excited states of furazan studied by DRM microwave spectroscopy. It should be noted that only the two CH-stretches $v_{1}$ and $v_{9}$ at $\sim 3100 \mathrm{~cm}^{-1}$ are omitted from this diagram. (The notations $2 \mathrm{C}^{13}, 2 \mathrm{~N}^{15}$ and $2 \mathrm{D}$ refer to molecular forms with the isotopes in either of the two equivalent positions.) 
gram a scale of vibrational energy $\left(\mathrm{in}^{-1}\right.$ ) is drawn on the left hand side, and the population of states according to the Boltzmann law is given on the right hand side for a temperature of $T=300 \mathrm{~K}$. Along that same scale are indicated the natural abundances of the isotopomers involving ${ }^{13} \mathrm{C},{ }^{15} \mathrm{~N},{ }^{18} \mathrm{O}$ and deuterium (D). This suggests that when the sensitivity of a (DRM) spectrometer is sufficient to allow the detection [12] of a deuterium form of a molecule in its natural abundance of $0.015 \%$, it should also be possible - at least in principle - to detect the pure rotation spectra of vibrationally excited states with comparably small populations, i.e. with vibrational energies in the range of $1800 \mathrm{~cm}^{-1}$. - The manifolds of purely rotational energy levels of excited states are symbolised in Fig. 2 as "sails", drawn at the appropriate position of vibrational energy and with a height that represents the rotational energy of levels. They are labelled by the microwave designation of the rotation spectra. The small numbers at the top of the "sails" give the highest $J$-value of observed transitions. The horizontal shading of the sails is meant to indicate rotational levels, and is replaced by "wavy" lines where perturbations have been noticed. The curved edge of the sails is intended to indicate the decreasing Boltzmann population of levels on account of their rotational energy. Unfortunately, neither the Boltzmann function nor the density and position of rotational levels can be drawn to scale. - Across the top of the diagram, finally, the IR designations of vibrational modes are given, with separate columns inserted for overtone and combination levels. The designation ' $1+7$ ', for example, is thus readily recognised as denoting the combination state $\left(v_{15}=1, v_{12}=1\right)$.

\section{e) Appraisal of Quantitative Results}

In the absence of degeneracies the dependence of effective rotational constants on vibrational excitation is commonly approximated [13] as

$$
R_{\text {effective }}=R_{\mathrm{c}}-\sum \alpha_{i}\left(n_{i}+1 / 2\right),
$$

where $R_{\mathrm{c}}$ is the (hypothetical) rotational constant of the molecule "frozen" in its equilibrium configuration, and the $\alpha_{i}$ are the so-called rotation-vibration constants for the modes $i$.

Extensive use of this approximation has been made during the experimental stage of this study. It allowed the prediction of the rotational constants (and hence of the spectra) of vibrational overtone and combina- tion levels, whenever the $\alpha$-constants were known for the fundamentals in question. The experimental examination of such predictions (analysis of spectra) was essential not only for the continual assessment of the sensitivity of the spectrometer, but also for the identification of the states ' 10 ', '11' and '12' and their correlation with the IR data. These three satellites could be ascribed unequivocally to excitation of the modes $v_{11}$, $v_{3}$ and $v_{2}$ only after the overtone and combination spectra (see Fig. 2) had been eliminated. Although details from that work are omitted from the present paper for the sake of brevity, satisfactory agreement of experimental findings with the above approximation was noted throughout. This justifies the extraction of the $\alpha_{i}$-constants in Table 2 as differences between corresponding constants of each fundamental level and the G.S. rotational constants:

$$
\alpha_{\mathrm{R}, i}=R_{\mathrm{G} . \mathrm{S} .}-R_{i, n_{i}=1} \quad(R=A, B, C) .
$$

Zero-point vibration contributes for each mode $i$ half that amount to the equilibrium constants $R_{\mathrm{e}}$ to give rise to the effective rotational constants of the G.S. - Hence, if the $\alpha_{i}$-values were available for all 15 normal modes of furazan, the equilibrium rotational constants $R_{\mathrm{e}}$ could be calculated from the effective constants $R_{\mathrm{G} . S .}$ of the ground state.

While the pure rotation spectra associated with excitation of the two $\mathrm{C}-\mathrm{H}$ stretching vibrations $v_{1}$ and $v_{9}$ at $\sim 3150 \mathrm{~cm}^{-1}$ cannot be considered for investigation by DRM spectroscopy on account to the present limitation in sensitivity, a satellite spectrum ' 13 ', assignable to excitation of the mode $v_{10}$ at $\sim 1541 \mathrm{~cm}^{-1}$ (approximate description: antisymmetric $\mathrm{C}=\mathrm{N}$ stretch) has not been identified in this study. Furthermore, the rotation-vibration constants $\alpha_{R, 3}$ and $\alpha_{R, 4}$, calculated in Table 2 from the approximate and perturbed rotational constants for the Coriolis dyad $\left(v_{13}, v_{14}\right)$ appear too inaccurate and may be principally inadequate for the task in hand. Hence, vibrational contributions from only 10 normal modes can be extracted from the effective G.S. constants to yield an approximation, $R_{\mathrm{e}}^{(10)}$, to the equilibrium constants of furazan. - This calculation is presented in Table 6.

This table shows that all three constants $R_{\mathrm{c}}^{(10)}=$ $R_{\mathrm{G} . \mathrm{s} .}+\sum \alpha_{\mathrm{R}, i} / 2$ (which correspond to a furazan molecule with only five normal modes (i.e. $v_{13}, v_{14}, v_{10}, v_{9}$, and $v_{1}$ ) with 10 modes "frozen") are larger than the observed $R_{\mathrm{G} . s .}$. The corresponding moments, $I_{\mathrm{e}}^{(10)}$, lead to a larger inertia defect than has been observed 
Table 6. Contributions from individual modes to the effective rotational constants (in $\mathrm{MHz}$ ) and to the inertia defect (in $u \AA^{2}$ ) in furazan.

\begin{tabular}{|c||cccc|}
\hline Mode $\underline{i}$ & $a_{\mathrm{A}, \mathrm{i}} / 2$ & $\alpha_{\mathrm{B}, \mathrm{i}} / 2$ & $\alpha_{\mathrm{C}, \mathrm{i}} / 2$ & $\delta(\mathrm{ID})_{\mathrm{i}} / 2$ \\
\hline 1 & +14.84 & +28.80 & 0.00 & -0.2249 \\
2 & +10.47 & +17.26 & -0.71 & -0.1558 \\
$(3)$ & $(+30.7)$ & $(-118.7)$ & $(-10.3)$ & $(+0.275)$ \\
$(4)$ & $(+23.5)$ & $(+136.9)$ & $(+13.6)$ & $(-0.596)$ \\
5 & -9.62 & +30.24 & +11.08 & +0.1031 \\
6 & +12.85 & -11.16 & -0.43 & -0.0079 \\
7 & -7.15 & -2.29 & +6.27 & +0.1713 \\
8 & -4.11 & +6.31 & -1.85 & -0.0519 \\
9 & -5.79 & +3.66 & -2.90 & -0.0511 \\
10 & -4.34 & -7.25 & +2.25 & +0.1043 \\
11 & +4.76 & +8.64 & +4.59 & +0.0234 \\
12 & +11.83 & +6.90 & +4.95 & +0.0073 \\
\hline${ }^{(10)}$ & +23.74 & +81.11 & +23.25 & -0.0823 \\
\hline $\mathrm{R}_{\mathrm{e}}^{(10)}$ & 10480.95 & 9762.76 & 5046.95 & \\
$\mathrm{I}_{\mathrm{e}}^{(10)}$ & 48.2185 & 51.7657 & 100.1349 & \\
$(\mathrm{ID})$ & & & & \\
\hline
\end{tabular}

for the G.S. Those five modes, for which no information is available, must therefore be judged to contribute rather significantly $\left(+0.151 \mathrm{u} \AA^{2}\right)$ to the inertia defect, which has to be zero for the equilibrium configuration. This result is confirmed by consideration of the inertia defect contributions from individual modes (right hand column of Table 6): The ten modes analysed are seen to contribute $-0.082 \mathrm{u} \AA^{2}$ together, while the G.S. was observed to have an inertia defect of $+0.071 \mathrm{u} \AA^{2}$. It follows again that the five modes, for which no or no reliable data are presently available, must contribute $+0.153 \mathrm{u} \AA^{2}$ to the inertia defect of furazan. This unexpectedly large contribution makes a future experimental examination of the three unstudied vibrations $v_{10}, v_{9}$, and $v_{1}$ highly desirable. Such work (by FT-IR spectroscopy) would be expected to resolve the present question whether the large residual inertia defect arises primarily from the comparatively low vibrational modes ' 3 ' and ' 4 ' (Coriolis dyad $v_{13}, v_{14}$ ) or from the $\mathrm{C}-\mathrm{H}$ stretching modes $v_{1}$ and $v_{9}$ at about $3100 \mathrm{~cm}^{-1}$.

\section{Discussion}

The present microwave study establishes molecular information which could not have been obtained with the techniques of either rotational or vibrational spectroscopy of the early 1960 s. Both fields however have progressed significantly since then.

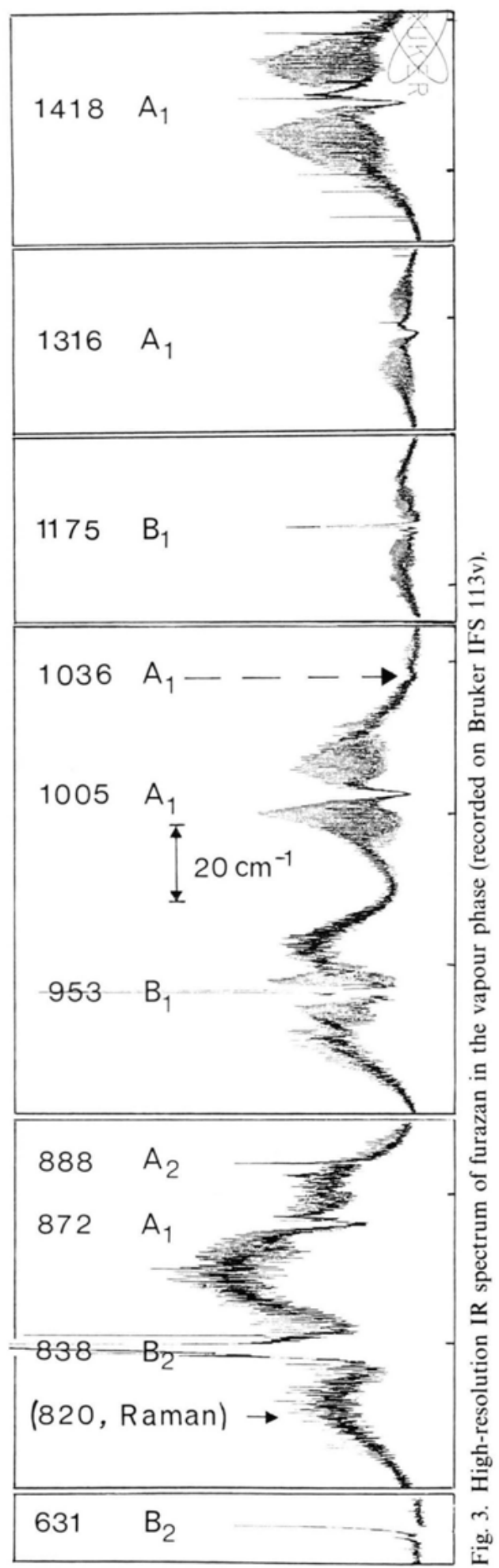


One experimental advance since that time, which we have been exploiting during the past two decades, is that of Double Resonance Modulation, which was introduced into microwave spectroscopy by E. Bright Wilson, Jr. and collaborators in 1966 [2]. We see the paramount advantage of this modulation technique over Stark effect modulation, introduced also by E. Bright Wilson, Jr. some 30 years earlier [3], in its extreme molecular selectivity. As has been demonstrated on a number of examples (see [1 a] or [12] for further references), this selectivity, when coupled with good spectroscopic sensitivity, allows the spectra of quite rare isotopomers to be studied in their natural abundances. As shown in the present paper, it also permits the detection and analysis of the pure rotation spectra of molecules in vibrationally excited states up to vibrational energies of $\sim 1500 \mathrm{~cm}^{-1}$. Hence it allows the identification and interpretation of nearly all absorptions detected in microwave spectroscopy and the exploitation of the major portion of molecular information commonly on offer.

Developments in vibrational spectroscopy during the same two decades have resulted in the replacement of low-resolution instruments by the high-resolution interferometers (with subsequent Fourier transformation of the interferogram) of today. As a result, molecular vibration spectra can now be recorded under resolutions of $10^{-3} \mathrm{~cm}^{-1}$ and, provided the rotational fine structure of such (resolved) IR bands can be unravelled, vibrational frequencies may be determined with the same degree of accuracy.

Figure 3 shows the vibrational spectrum of furazan in the range from $600 \mathrm{~cm}^{-1}$ to $1440 \mathrm{~cm}^{-1}$, taken on a commercial, modern IR instrument (Bruker, IFS 113v) with a resolution of $0.03 \mathrm{~cm}^{-1}$ at a sample pressure of 15 Torr. Inspection shows that 10 of the 12 fundamentals rotationally analysed in the present study are readily detectable and most likely well resolvable in the IR spectrum. The $A_{1}$ mode at $1036 \mathrm{~cm}^{-1}$ (arrow) is, admittedly, very weak and the $A_{2}$ mode at $636 \mathrm{~cm}^{-1}$ is duely missing (IR inactive). The $\mathrm{A}_{2}$ mode at $888 \mathrm{~cm}^{-1}$, on the other hand, is detectable presumably as a result of perturbations. No indication of the $B_{1}$ mode at $820 \mathrm{~cm}^{-1}$ is discernable in the vapour phase spectrum of Fig. 3, but the frequency of that vibration has been determined in the spectrum of the liquid and by Raman spectroscopy [6].

The improvement of IR resolution justifies the hope that all these bands can be satisfactorily resolved and analysed in the near future and that their centre frequencies can be determined to $\pm 0.001 \mathrm{~cm}^{-1}$ or better. This task should be greatly facilitated through the rotational information established here. - It does not seem too optimistic an outlook that FT-IR spectroscopy will permit the mode at $1541 \mathrm{~cm}^{-1}\left(\mathrm{~B}_{1}\right.$, antisymmetric $\mathrm{C}=\mathrm{N}$ stretch) and the two $\mathrm{C}-\mathrm{H}$ stretches at $3150 \mathrm{~cm}^{-1}$ to be rotationally analysed and to have their inertia defect contributions and their vibration frequencies determined accurately. This information would allow the contribution from vibrational motion to the G.S. moments of inertia to be determined for all modes. At the same time, accurate knowledge of all 15 fundamental frequencies would allow an improved determination of the force field within this molecule.

Such internal potentials, if established for a sufficient number of substances, might in the long run provide a more detailed understanding of chemical reactivity than has been available in the past.

\section{Acknowledgements}

The author wishes to express his sincere gratitude to Dr. D. Christen of the Universität Tübingen for encouragement and invaluable discussions on the present work. He is equally indebted to "Bruker Analytische Meßtechnik", in particular to Dr. G. Zachmann, for providing him (on the occasion of one of his private visits to Rheinstetten) with the IR trace from which Fig. 3 is constructed. Dr. Brenda P. Winnewisser of the Universität Gießen has been so kind as to read critically the manuscript and has helped to eliminate several "germanisms". 
Measured transition frequencies (in MHz) of the G.S. and the first twelve fundamental levels in furazan.

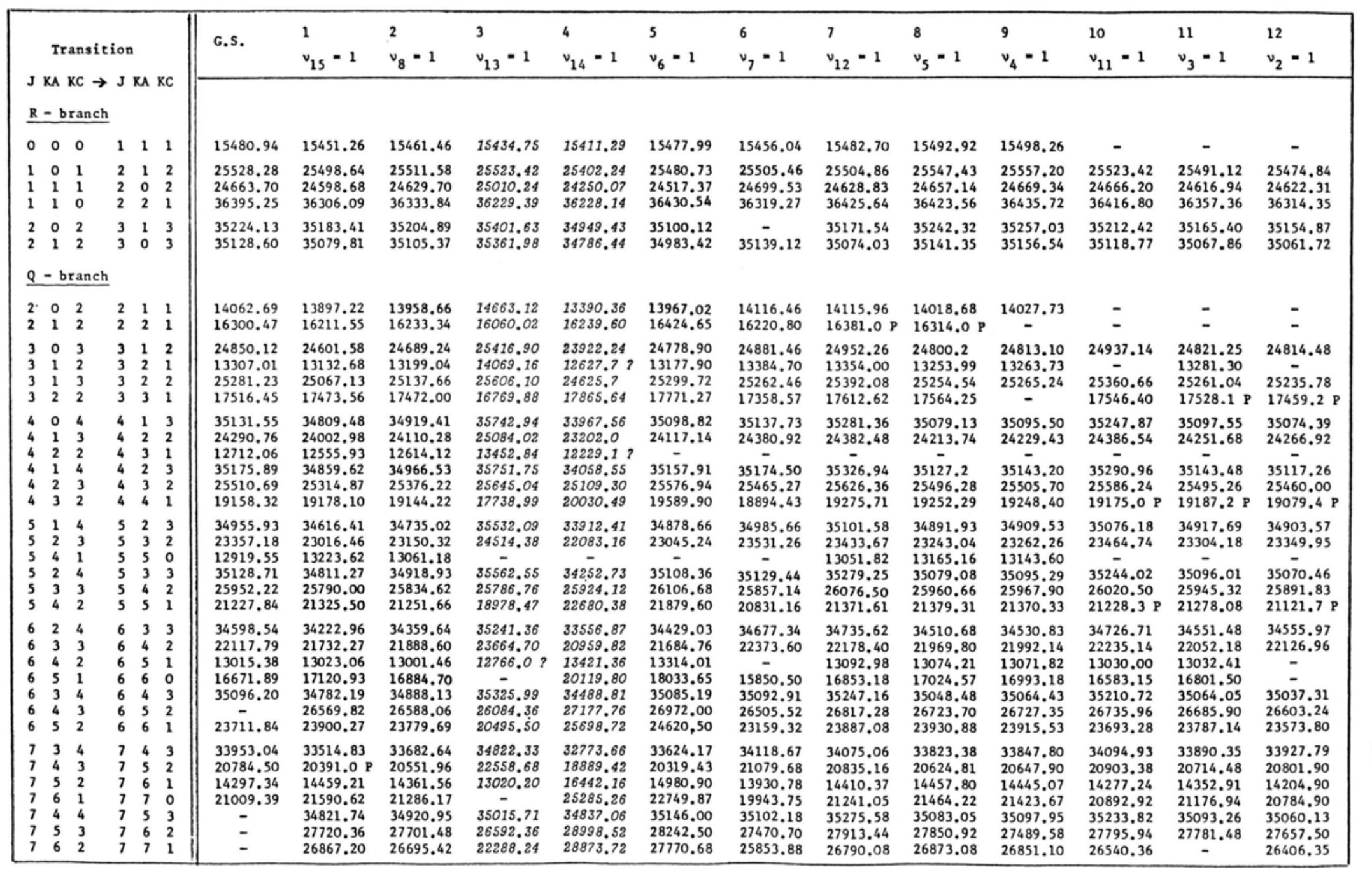




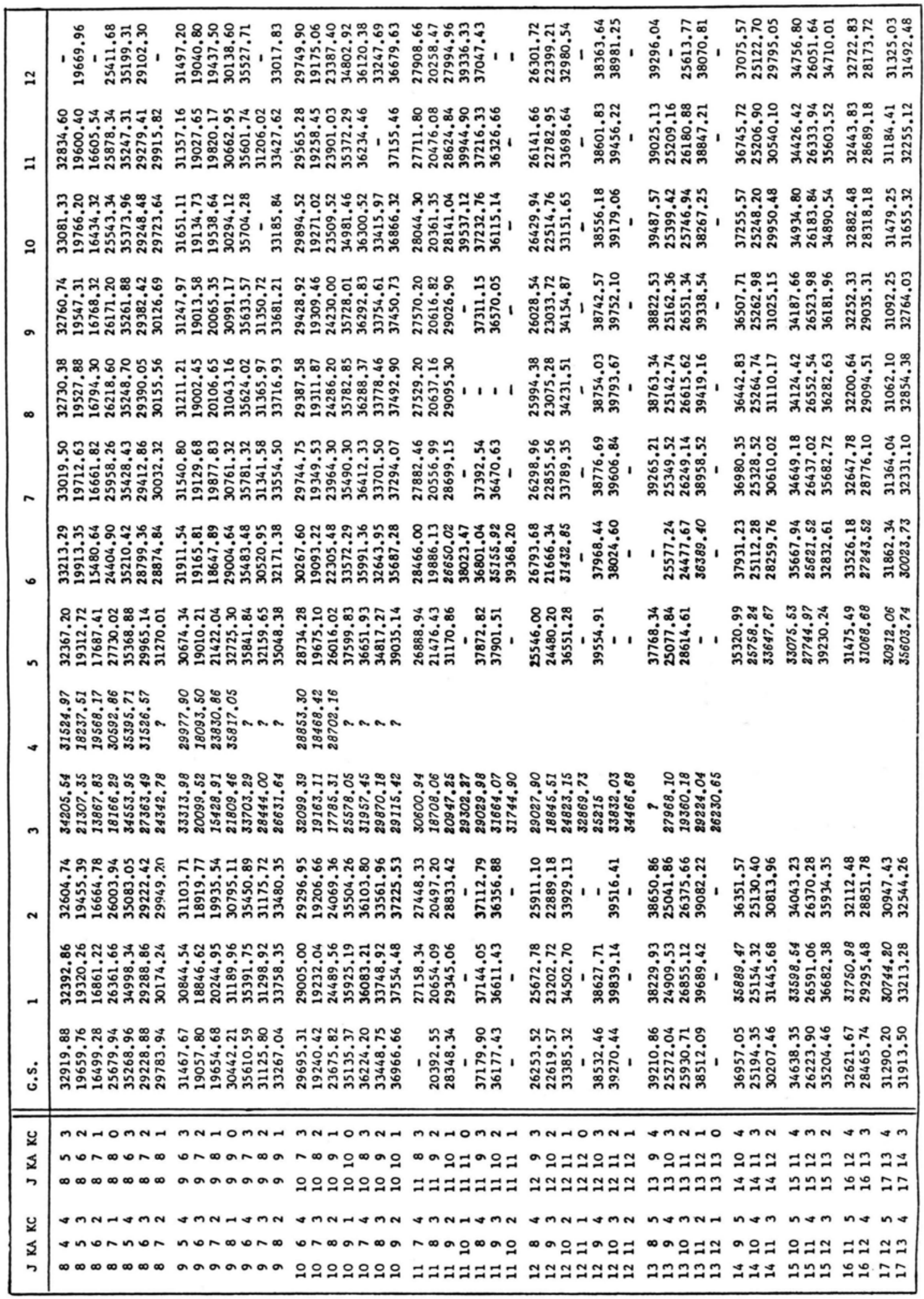


[1] O. L. Stiefvater, Z. Naturforsch. a) 43a, 597 (1988); b) 43a, 607 (1988); c) 30a, 1742 (1975); d) 30a, 1756 (1975); e) 33a, 1511 (1978).

[2] R. C. Woods III, A. M. Ronn, and E. B. Wilson, Jr., Rev. Sci Instrum. 37, 927 (1966).

[3] R. H. Hughes and E. B. Wilson, Jr., Phys. Rev. 71, 562 (1947).

[4] G. Herzberg, Infrared and Raman Spectra of Polyatomic Molecules, Van Nostrand Reinhold Co., New York 1945.

[5] E. Borello, A. Zechina, and E. Guglielminotti, Gazz. Chem. Ital. 96, 852 (1966).

[6] G. Sbrana, M. Ginnaneschi, and M. P. Marzocchi, Spectrochim. Acta 23 A, 1757 (1967).

[7] D. H. Christensen and O. F. Nielsen, J. Mol. Spectroscopy $\mathbf{2 4}, 477$ (1967)

[8] D. H. Christensen, P. W. Jensen, J. T. Nielsen, and O. F. Nielsen, Spectrochim. Acta 29 A, 1393 (1973).

[9] A. S. Esbitt and E. B. Wilson, Jr., Rev. Sci. Instrum. 34, 901 (1963).

[10] E. B. Wilson, Jr., J. C. Decius, and P. C. Cross, Molecular Vibrations, McGraw-Hill Book Co., New York 1955.

[11] C. H. Townes and A. L. Schawlow, Microwave Spectroscopy, McGraw-Hill Book Co., New York 1955.

[12] O. L. Stiefvater, J. Chem. Phys. 63, 2560 (1975).

[13] W. Gordy and R. L. Cook, Microwave Molecular Spectra, Interscience Publishers 1970. 